\title{
The Open Approach to a Supracondylar Humerus Fracture
}

Aditi S. Majumdar, MD, MS and Andrea S. Bauer, MD

Boston Children's Hospital, Boston, MA

Correspondence to: Andrea S. Bauer, MD; 300 Longwood Ave., HUN 213, Boston, MA 02115, E-mail: andrea.bauer@childrens.harvard.edu

Received: January 19, 2022; Accepted: January 19, 2022; Published: February 1, 2022

DOI: 10.55275/JPOSNA-2022-0020

\begin{abstract}
:
Open approach to a supracondylar humerus fracture may be needed for open fractures, vascular compromise, and most commonly, irreducible fractures refractory to closed management. Gartland types III and IV fractures as well as flexion-type fractures are more likely to require an open technique. Vascular compromise can be overt as is the case of a white pulseless extremity or more subtle as is with the pink pulseless extremity. The use of Doppler signals intraoperatively can be helpful to distinguish which "pink pulseless" cases warrant open exploration. Intraoperative signs of irreducibility include persistent gapping at the fracture site, loss of Baumann's angle, and discontinuous columns on oblique views. In the scenario an open technique may be necessary, our recommended setup involves positioning of the patient supine on an operating table with a radiolucent hand table attached. An anterior approach to the antecubital fossa is both cosmetic and extensile. This is our preferred approach for irreducible extensiontype fractures, suspected interposition of brachialis muscle and fascia, median nerve sensory deficits, and vascular exploration. The medial approach is best for flexion-type fractures, ulnar nerve entrapment, extension-type fractures with posterolateral displacement, and oblique or unstable fractures necessitating medial pinning. Postoperative treatment is similar after open technique as is for closed pinning of a supracondylar humerus fracture.
\end{abstract}

\section{Key Concepts:}

- Beyond open fractures, consider an open approach for vascular compromise and irreducible fractures.

- Doppler signals can help differentiate between a pulseless extremity that is perfused and one that requires further vascular intervention.

- In cases when an open approach may be needed, consider OR setup using a radiolucent hand table. 
- Anterior approach is best for irreducible extension-type fractures and is extensile in case neurovascular repair/ reconstruction is warranted.

- Medial approach is best for irreducible flexion-type fractures as well as the placement of a medial pin.

\section{Introduction}

Supracondylar humerus fractures represent the most common pediatric elbow fracture, predominating in children 3-6 years of age and constituting approximately $15 \%$ of all pediatric fractures. ${ }^{1-3}$ Consistent with the recommended standard of care, the vast majority of operative supracondylar humerus fractures are treated with closed reduction and percutaneous pinning (CRPP) with select indications for open treatment. The estimated rate of supracondylar humerus fractures requiring open reduction is $12.7 \%,{ }^{4}$ with a mere $1.7 \%$ rate for closed injuries. ${ }^{5}$ Classic indications for an open technique include open fractures, vascular compromise, and most commonly, irreducible fractures refractory to closed management. ${ }^{6}$ Recent studies suggest that indications for open surgery may be further expanded to include delay in presentation ( $>24$ hours post injury), ${ }^{7}$ associated median nerve sensory deficits, ${ }^{8}$ and evolving compartment syndrome, in which direct visualization and access to the antecubital fossa may be desired. Due to limited consensus regarding optimal surgical approach, infrequent and wide variability in open treatment thresholds, and inherently different considerations to each indication, we aim to precisely summarize operative indications for open treatment and elucidate safe open technique principles.

\section{Background}

\section{Classification}

To understand the basis of open management, it is important to first understand supracondylar humerus fracture morphology, severity, and complexity. The commonly used Gartland Classification, derived on radiographic parameters, provides an effective, universal language that is both easily interpreted and reliably anticipates injury features and necessary treatment (Figure 1).
Extension-type fractures (97-98.8\%) encompass the large majority of fractures, followed distantly by the flexion-type variant (1.2-3\%). ${ }^{2,9,10}$ In general, higher severity fractures (extension-types III and IV and flexion-type supracondylar humerus fractures) correspond with higher energy, unstable injuries and are more likely to require open treatment than extensiontypes I and II. ${ }^{9,10}$ Flexion-type fractures are far more likely to require open reduction in comparison to extension-type injuries, exacerbated in the presence of an ulnar nerve palsy. ${ }^{11}$

\section{Relevant Anatomy}

In addition to fracture type, supracondylar humerus fractures can be further defined by displacement patterns in the sagittal and coronal planes (Figure 2).

Knowledge of the displacement pattern is clinically pertinent in understanding potential impediments to reduction as well as to distinguishing whether a neurovascular injury is likely a penetrating versus a tethering injury due to the proximal metaphyseal fragment. The ulnar nerve, coursing directly posterior to the medial epicondyle within the cubital tunnel, is at greatest risk with flexion-type fractures and at added risk of iatrogenic injury with medial pin fixation, given its potential instability with varying degrees of elbow flexion. Anterior ulnar nerve subluxation (27.5\%) and dislocation (near 10\%) over the medial epicondyle is frequent in the non-pathologic pediatric elbow. ${ }^{12}$ The radial nerve, traversing posterior to anterior immediately proximal to the olecranon fossa, is at greatest risk with posteromedial fracture displacement in extension-type fractures. The brachial artery and median nerve, running parallel, deep, and medial to the biceps tendon at the 
A

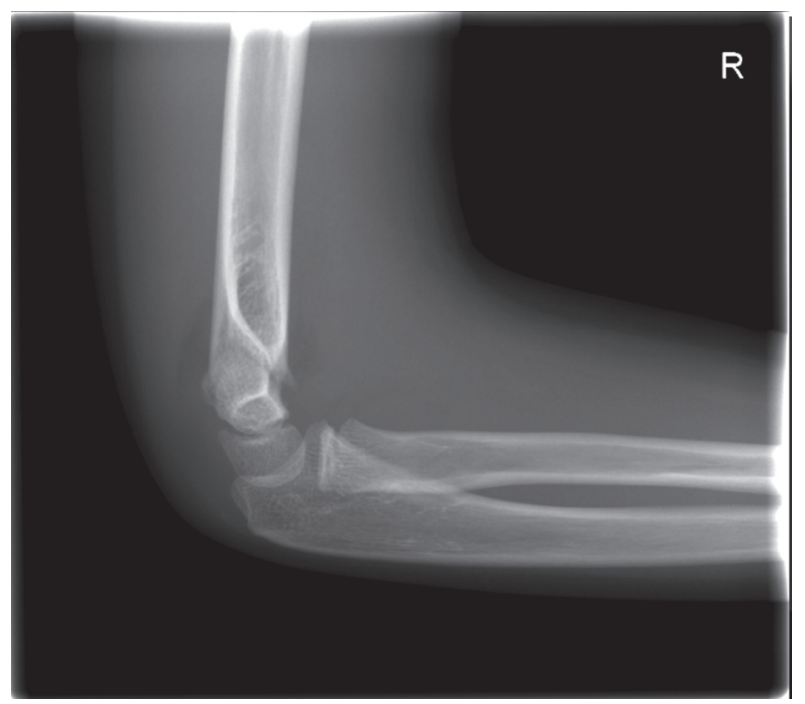

C

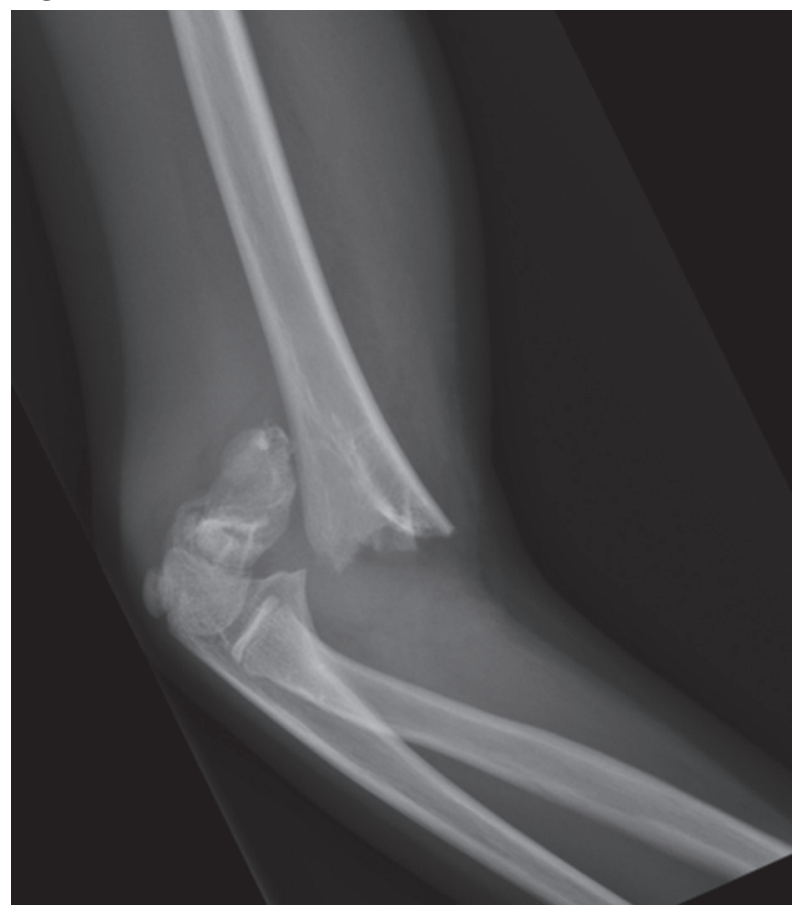

B

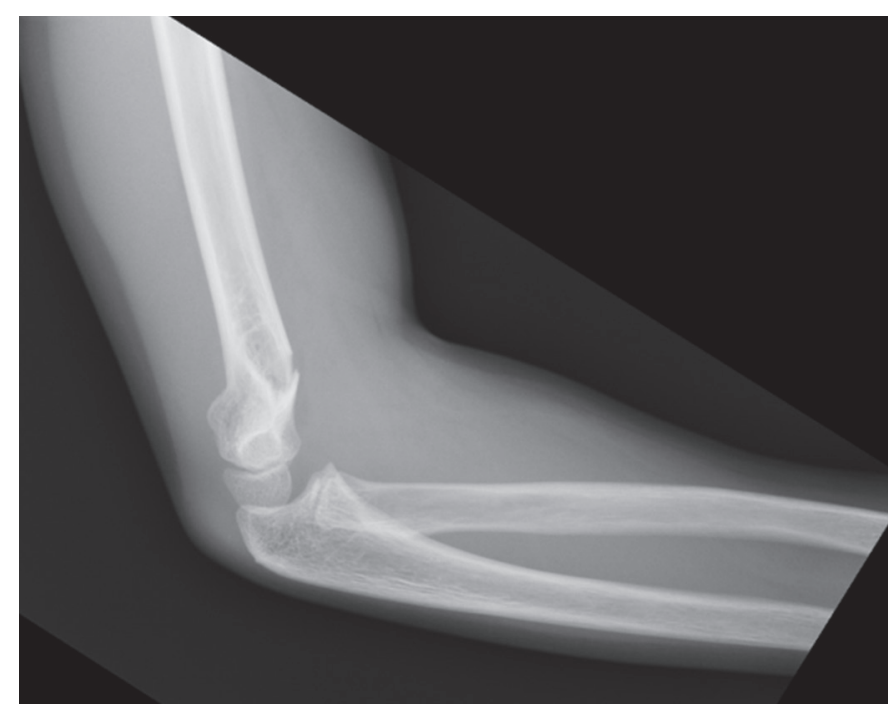

D

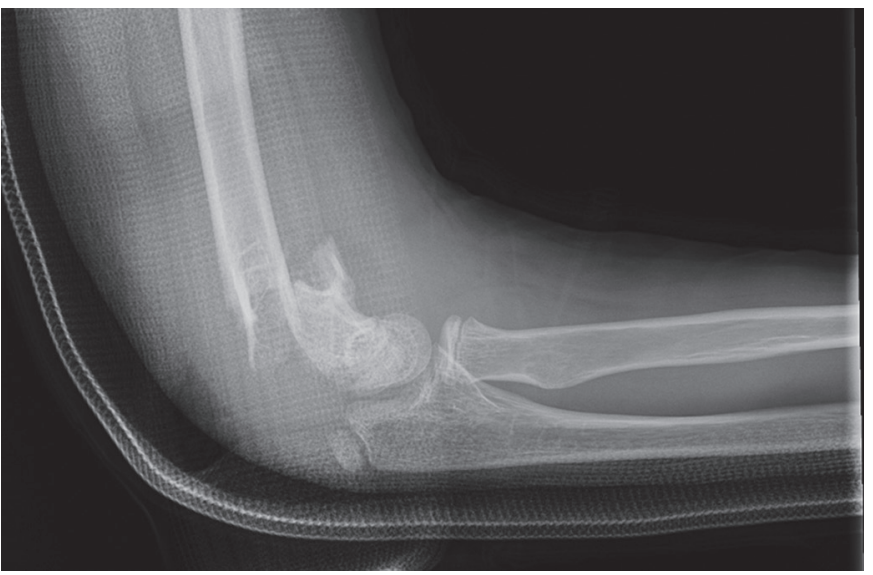

Figure 1. (A) Type 1: nondisplaced. (B) Type II: anterior humeral line posterior to capitellum, in varying amounts of extension angulation, posterior humerus intact. (C) Type III: distal fragment displaced, complete cortical disruption. (D) Flexion type, with distal fragment anteriorly displaced. Gartland type IV fractures are diagnosed intraoperatively based on global instability and can appear preoperatively as either an extension-type III fracture or a flexion-type fracture.

antecubital fossa, are most vulnerable with severely displaced posterolateral, extension-type fractures. A suspected or identified injury of either the nerve or the artery should alert attention to its counterpart given intimate proximity. ${ }^{8,13}$ The anterior interosseous nerve
(AIN) is the most commonly injured nerve associated with extension-type supracondylar humerus fractures (34.1\% of extension-type neuropraxias), ${ }^{14}$ however, injury to the AIN has not been shown to be prognostic of fracture severity (Table 1). 

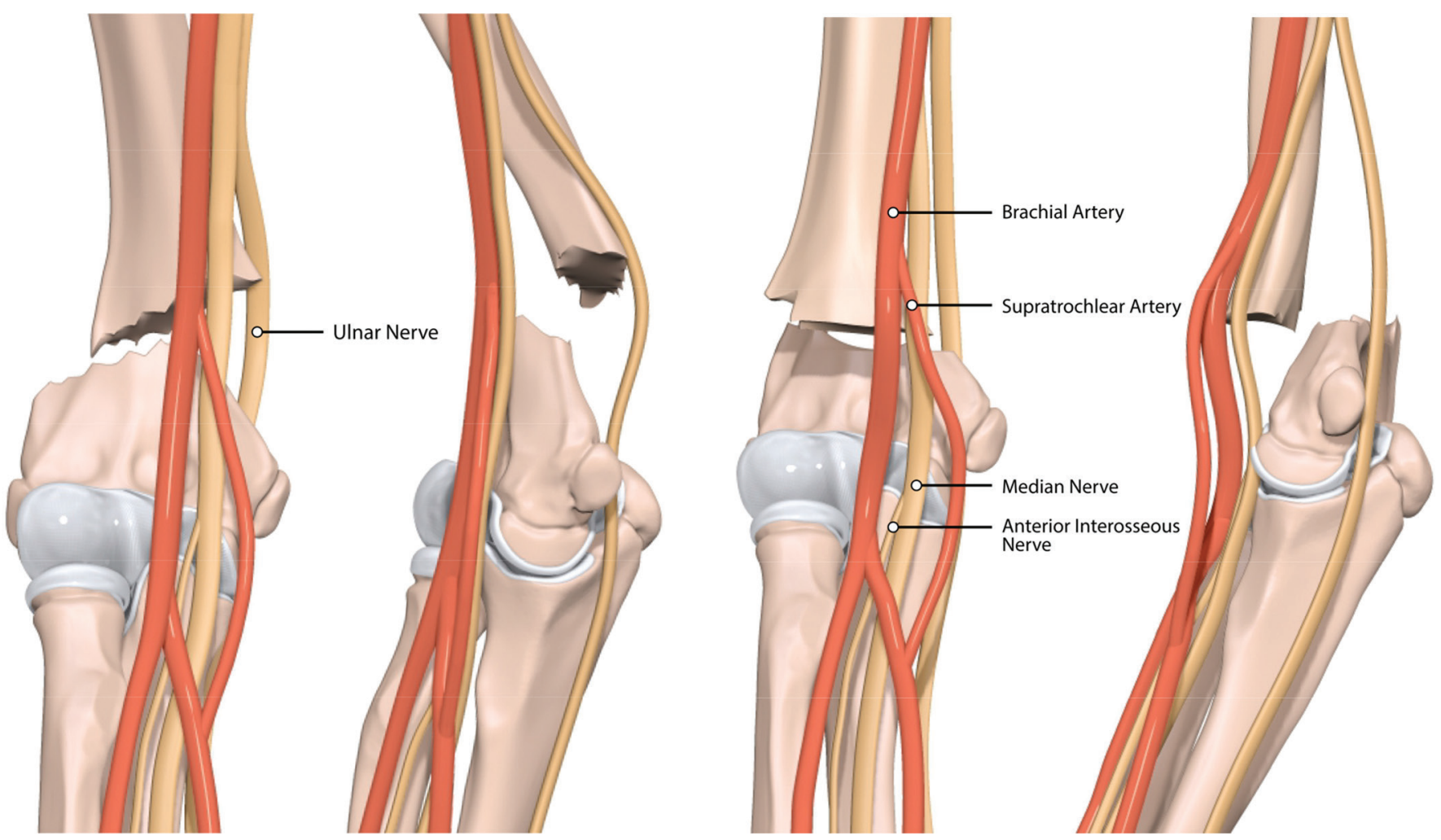

Figure 2. Examples of displaced flexion-type supracondylar fracture (left two figures) and extension-type supracondylar fracture (right two figures) with overlying neurovascular structures.

\section{Periosteum, joint capsule, and brachialis muscle and} fascia are the most common impediments to anatomic reduction with extension-type supracondylar humerus fractures. Presence of antebrachial ecchymosis and/or the "pucker sign" can alert the surgeon to a higher likelihood of interposed tissue which may require open extrication. Conversely, it is important to remember that an intact periosteal sleeve may act as a powerful indirect aid to fracture reduction and is optimally left maintained. The surgeon should consider where the intact periosteum may be during open surgical approach and dissection to use the intact periosteum to advantage and not disrupt it unnecessarily.

\section{Physical Exam Findings}

A thorough preoperative neurologic and vascular examination should be conducted and documented prior to initiation of treatment, although this is undoubtedly a challenge in very young patients. Accurate, timely vascular assessment of the involved extremity is critical and optimally encapsulates reproducible subjective and objective measures, including palpation of the distal radial pulse, clinical evaluation of perfusion (capillary refill, skin temperature and color, pulse oximetry), and Doppler signal of the distal radial pulse.

A "pucker sign" or brachialis sign occurs as a result of proximal fracture fragment penetration through the brachialis muscle and anterior fascia into subcutaneous tissue and is indicative of significant soft tissue injury from an extension-type fracture (Figure 3).

\section{Indications For Open Treatment}

\section{Open Fractures}

Open supracondylar humerus fractures are relatively rare, representing only $1-2.4 \%$ of all injuries. ${ }^{4,15,16}$ The vast majority of open supracondylar humeral injuries may be categorized as Gustilo-Anderson grade $1,{ }^{17}$ which are optimally treated with antibiotic prophylaxis and 


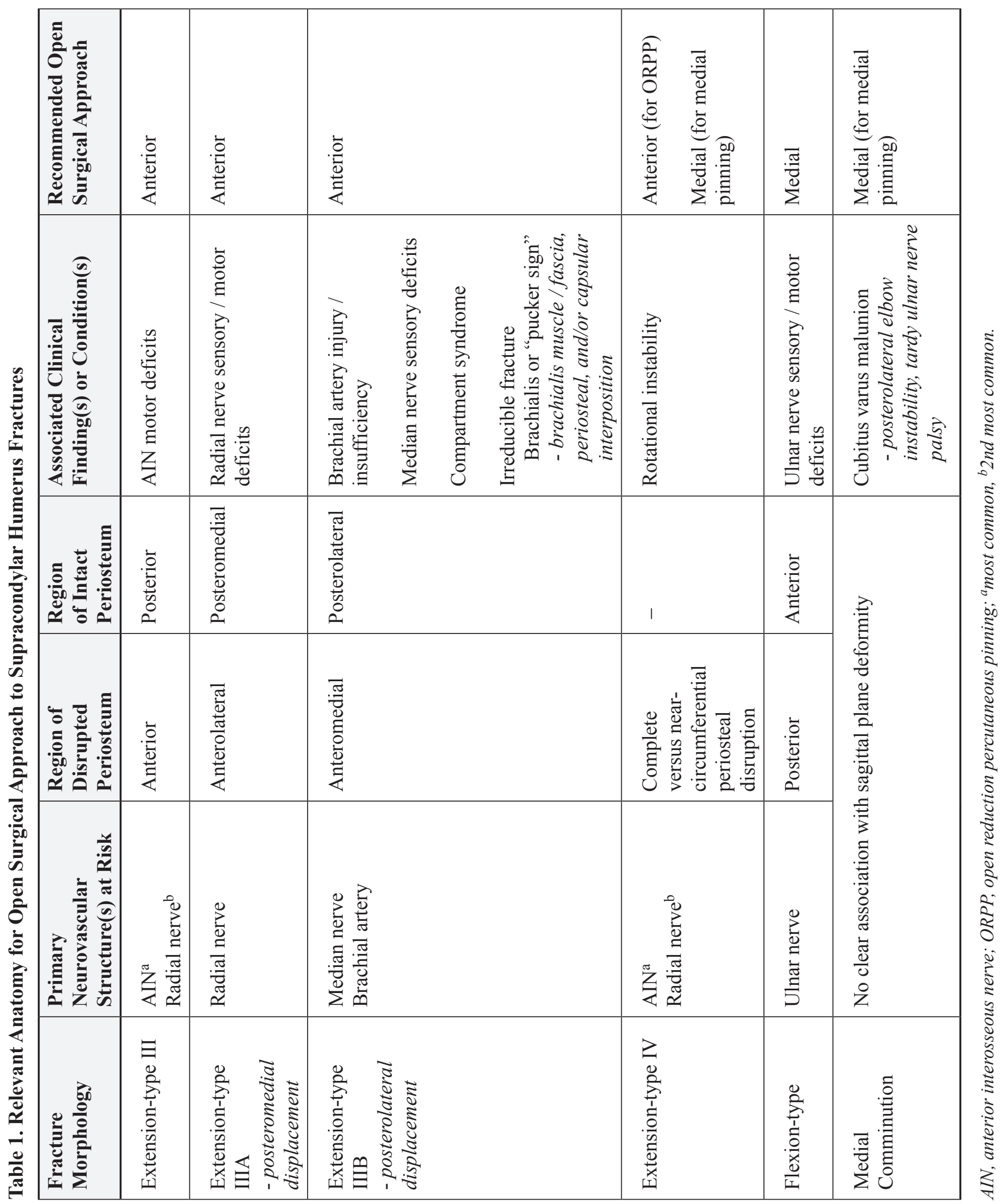


A

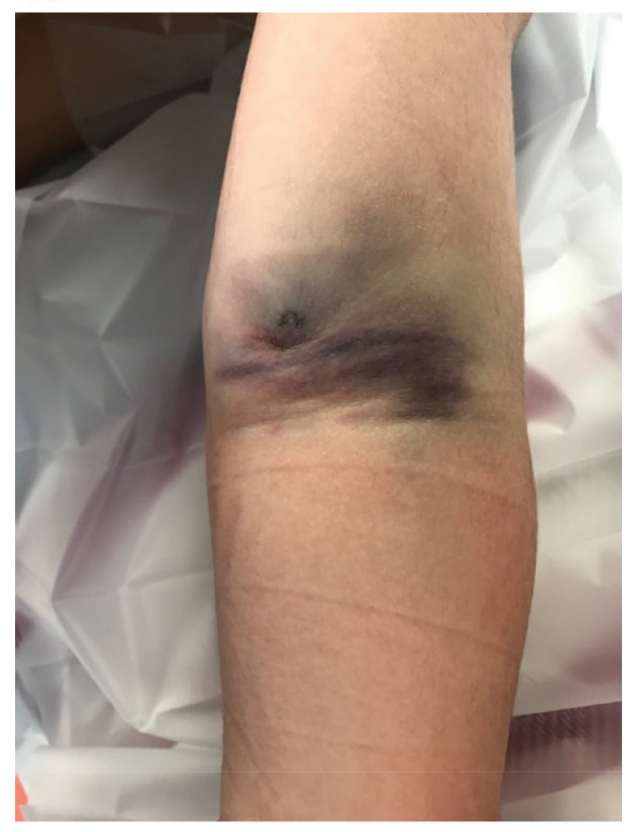

\section{B}

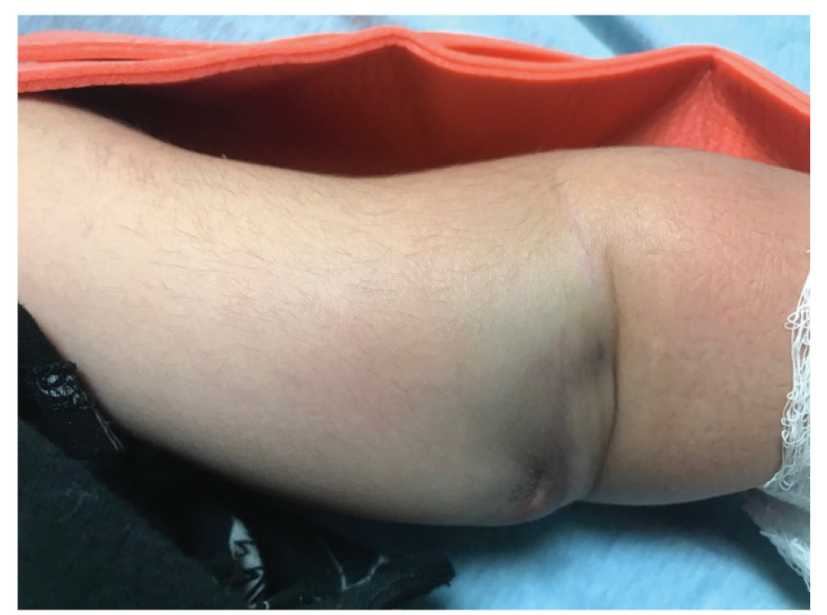

Figure 3. Clinical photographs of (A) antebrachial ecchymosis and (B) the antebrachial "pucker sign" (notice the deep skin creasing in the antecubital fossa region).

operative debridement to minimize secondary infection burden. However, these grade 1 open fractures can often be treated with closed reduction and pinning following appropriate debridement. Open supracondylar humerus fractures are often associated with higher energy mechanisms, yielding an overall higher incidence of ipsilateral forearm fractures ("floating elbow"), nerve injury, and potential need for vascular intervention in comparison to closed injuries. ${ }^{15}$ Because the open injury is typically readily apparent, open fractures are more likely to undergo an expedited trip to the operating room versus other similarly urgent injuries such as vascular insufficiency and compartment syndrome. ${ }^{15}$

\section{Nerve Injury}

The risk of neurovascular injury is elevated with greater degree of fractures displacement, with a suspected overall incidence of $49 \%{ }^{13}$ The true incidence of isolated neurologic injury may be underrepresented due to the innate challenges of an accurate nerve exam in the very young patient. Neurologic injuries (10-20\%) $)^{13,14}$ predominantly represent neuropraxia. As such, isolated nerve injuries do not in themselves necessitate open treatment, given spontaneous resolution in the majority of cases. Iatrogenic nerve injuries while infrequent $(\sim 6 \%),{ }^{2}$ primarily affect the ulnar nerve most often secondary to medial percutaneous pinning - a risk that can be mitigated with a mini medial open approach that provides direct visualization and protection to the nerve. ${ }^{14,18,19}$ Even with this open approach, the medial pin should be placed with the elbow in a semi-extended position, given the high rate of ulnar nerve subluxation in children as noted above. Early open reduction and antecubital fossa exploration should be considered in severely displaced fractures with associated vascular compromise and/or median nerve injury, given the legitimate risk of neurovascular entrapment at the fracture site. 8,20

\section{Vascular Insufficiency}

An associated or suspected vascular injury in the setting of supracondylar humerus fractures warrants emergent fracture reduction and skeletal stabilization. Anatomic fracture reduction is typically sufficient in restoring vascular flow (either directly or indirectly via abundant collateral flow), clinically appreciated by 
return of perfusion and distal pulses. The rate of true vascular compromise ranges widely in the literature (2-19\%) and may be attributed to antecubital hematoma (vascular occlusion) versus brachial artery stretch, vasospasm, entrapment, or transection. ${ }^{6,13,21,22}$ Longterm manifestations of persistent or prolonged limb vascular insufficiency are not insignificant and include cold intolerance, sensory changes, chronic pain, and/or weakness. ${ }^{23,24}$

A cool, "white pulseless hand" (distal limb ischemia, absent distal pulses) is indicative of severe vascular insufficiency and requires emergent reduction with potential for open surgical exploration and vascular reconstruction. If the "white pulseless hand" remains after appropriate closed reduction and pinning, open exploration is indicated. Controversy continues to surround optimal management of the "pink pulseless hand" (distal limb perfusion, absent distal pulses) following closed reduction, differing vastly from observation to urgent/emergent surgery with possible vascular intervention. Part of this controversy stems from the fact that the term "pink pulseless hand" does not clearly distinguish a transient brachial artery vasospasm from a true brachial artery injury and thus may be a misleading term. The clinical interpretation of a "pink hand" is susceptible to broad subjective variability with potential to assert different treatment pathways depending on surgeons' assessment. As such, we argue this term should not constitute the sole criteria for surgical decision-making. In addition to the diagnosis of vascular insufficiency being timely, accurate, and reproducible, we emphasize the need for simple, accessible, objective criteria such as continuous pulse oximetry and Doppler assessment as essential components of the diagnostic process. An algorithm for including the use of Doppler signals in management of the "pink pulseless hand" is well depicted by Shah et al. ${ }^{22}$ and shown in Figure 4.

\section{Irreducible Fractures}

The rate of conversion from closed to open reduction secondary to irreducible fractures has been reported

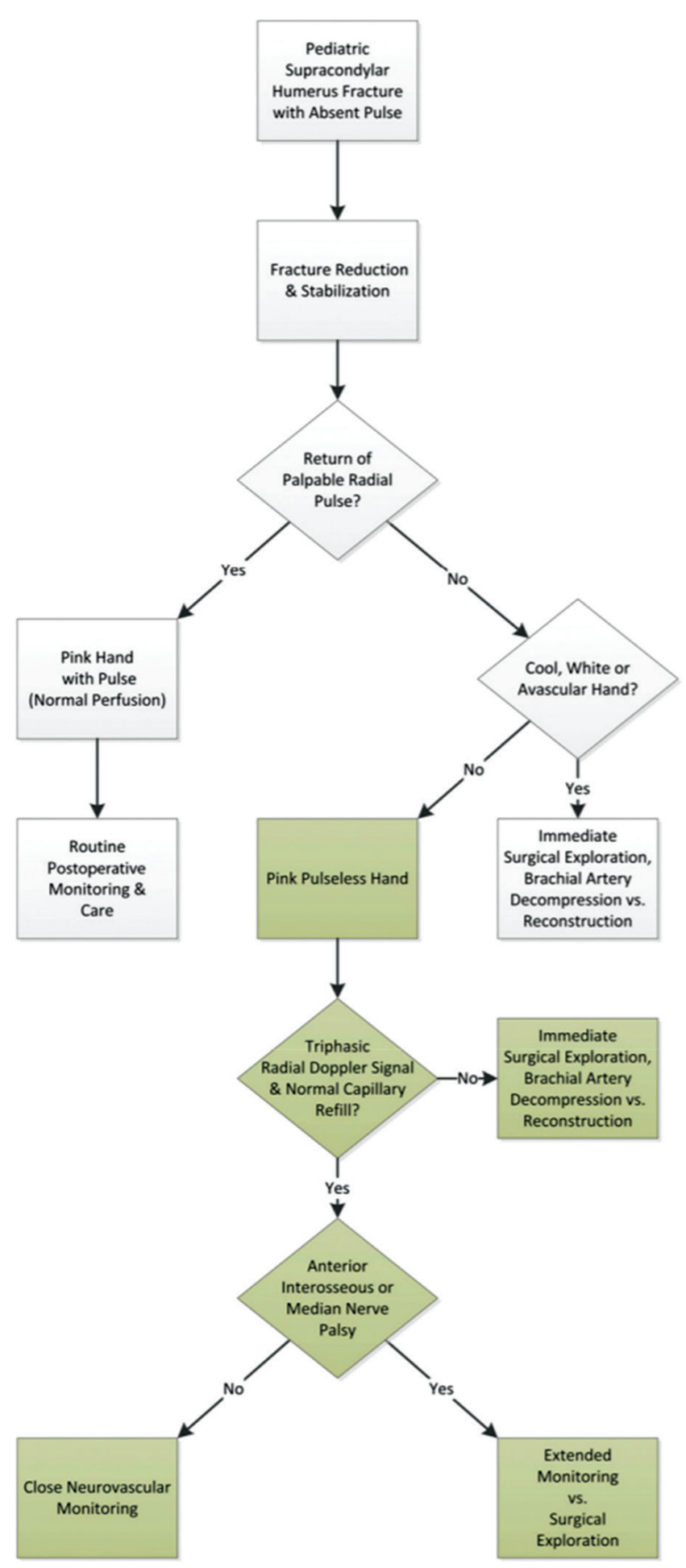

Figure 4. Algorithm for treatment of the "pink pulseless hand" associated with pediatric supracondylar humerus fracture. (Reprinted with permission from Shah AS, Waters PM, Bae DS. Treatment of the "Pink Pulseless Hand" in Pediatric Supracondylar Humerus Fractures. J Hand Surg Am Ed. 2013;38(7):1399-1403. doi:10.1016/j.jhsa.2013.03.047.) 
as ranging from $<3 \%$ to $46 \%{ }^{7}$ Anatomic reduction of the distal humerus fulfills the following radiographic criteria: bisection of the capitellum by anterior humeral line, intact medial and lateral columns on oblique views, and restoration of Baumann's angle near symmetric to contralateral elbow or 70-78 degrees. Failure of anatomic reduction prior to fixation may lead to cubitus varus malunion, reduction in elbow function, and loss of elbow motion. Irreducibility secondary to button-holing of the brachialis muscle and fascia by the proximal fragment, along with capsular interposition, can be predicted by a brachialis or pucker sign, posterolateral fracture displacement, and rotational instability. ${ }^{6}$ Radiographic features of irreducibility include persistent gapping at the fracture site, loss of Baumann's angle, and discontinuous columns on oblique views. Open reduction percutaneous pinning (ORPP) permits precise anatomic reduction by enabling direct extrication of interposed structures, decompression of hematoma, and subsequent fixation by direct fracture visualization.

\section{When to Consider Patient Transfer}

If open exploration is potentially warranted due to vascular compromise, we recommend early communication with an experienced hand or vascular surgeon prior to operative management. At our institution, the hand surgeon on call is alerted for all supracondylar humerus fractures that are pulseless prior to reduction, although the vast majority of these have successful return of pulses after appropriate closed reduction and pinning. For centers without immediate availability of a surgeon with expertise in vascular repair, it is reasonable to expedite transfer of the patient with a pulseless extremity to a higher care facility with safe, temporizing measures. Ideally, transfer agreements between referring and receiving institutions should be in place ahead of time for this scenario. Adjunctive measures to optimize distal perfusion in the preoperative waiting period or during patient transfer may include stabilizing the elbow in a slightly flexed position (20-40 degrees) to lessen tension on the brachial artery as well as keeping the extremity warm. A closed reduction prior to transfer to an outside facility may help to restore peripheral flow; however, this should be judiciously pursued and balanced with patient safety, prolonged ischemic time, and supplemental anesthesia requirements. Similarly, angiography is not typically warranted as the location and cause of the vascular injury are known, and prompt fracture reduction is paramount.

\section{Operative Planning}

\section{Fracture Management and Reduction Principles}

The majority ( $>95 \%$ ) of supracondylar humerus fracture can be effectively reduced through systematic closed reduction methods and techniques. The goal of initial fracture management should be anatomic reduction, as subsequent reduction attempts can lead to higher rates of secondary complications due to soft tissue edema, disruption of periosteal hinge, and iatrogenic repetitive trauma to fracture fragments. With more severe, potentially irreducible fractures, a provisional attempt at fracture reduction immediately after anesthesia induction may inform the surgical team about the probability of open reduction, necessary instrumentation and setup, and need for ancillary surgical support. Muscle paralysis is essential for atraumatic reduction in more severe, displaced injuries, and should be considered alongside the anticipated anesthesia plan.

\section{Operative Positioning and Setup}

In the scenario an open technique may be necessary, our recommended setup involves positioning of the patient supine on an operating table with a radiolucent hand table attached (Figure 5).

The bed is rotated 90 degrees. With rotationally unstable and flexion-type injuries, given that maintenance of reduction can be particularly challenging, we recommend fluoroscopic access parallel to the patient from the head or foot of the bed. This provides ability to rotate the $\mathrm{C}$-arm under the hand table for a cross-table lateral view without additional arm manipulation. In smaller children, the shoulder and head may need to partially reside on the hand table to facilitate complete visualization of the distal humerus and elbow. We recommend wide preparation of the affected extremity to level of the 


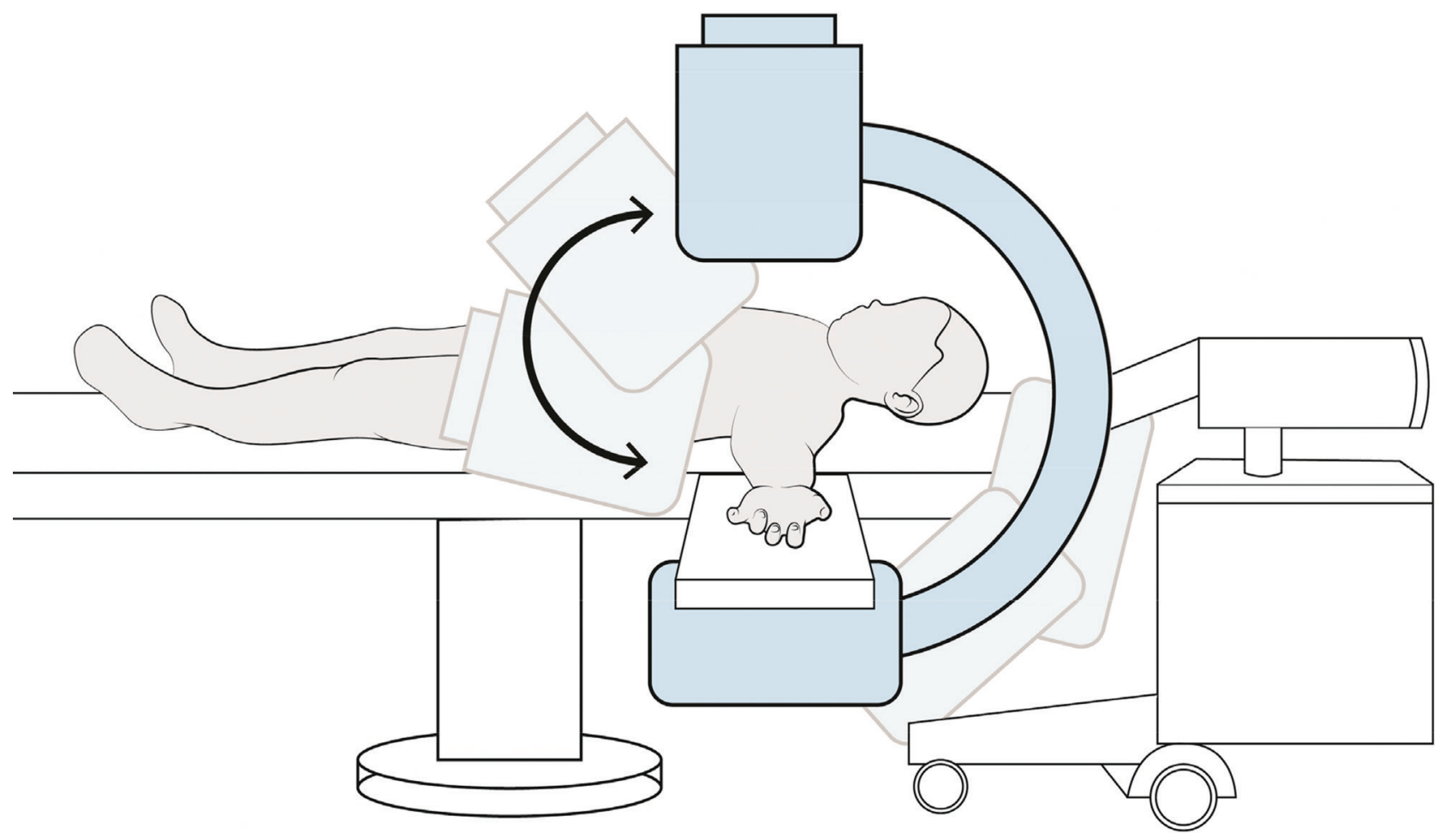

Figure 5. Operating room setup for opening a supracondylar humerus fracture.

axilla to facilitate fracture reduction as well as provide proximal access to the medial arm in the event vascular repair or reconstruction is warranted. We do not recommend routine use of a tourniquet, as this is unlikely to lend additional benefit and is more likely inhibitive to open exploration in an already small extremity. It should be noted, injury and trauma may distort the anatomic position of various structures, emphasizing the importance of anatomic relationships of vital relevant anatomy. Operative instruments for open reduction and microvascular repair should be readily available in anticipation of open exploration and potential vascular reconstruction. Doppler ultrasound and pulse oximetry should be immediately accessible for objective assessment of vascular status throughout the case.

\section{Closed Reduction Technique}

\section{Extension-Type}

For displaced extension-type fractures, prior to initiation of fracture reduction maneuvers, we recommend gentle, sustained longitudinal traction of the distal forearm with countertraction at the axilla and the elbow in a semi-extended position (10-30 degrees of flexion) for approximately 5 to 10 minutes. This traction maneuver gradually disengages the proximal humeral shaft from the anterior subcutaneous tissues, visibly reducing any tethering of skin anteriorly. In the setting of pronounced antecubital ecchymosis and puckering, intermittent supplementation with a "milking maneuver," can further release the proximal metaphyseal spike from its entrapment within the brachialis muscle and fascia.

In turn, subsequent larger reduction maneuvers may theoretically proceed in a more controlled fashion, requiring less force to restore sagittal plane alignment, and minimizing unnecessary insult to an intact periosteal hinge. This is particularly relevant in preventing conversion of a partially stable injury to unstable status as with an extension-type III to type IV or less desirable flexion-type. In typical sequence, coronal alignment should be attained prior to sagittal alignment. This is typically achieved through restoration of length, followed 
by medial or lateral translation as dictated by fracture displacement. Utilizing intact periosteum as a reduction aid, the distal fragment is influenced by the surgeon's thumb on the appropriate side and met with counter pressure on the opposing side. Alternatively, lateral translation can be achieved through forearm supination and pronation with the arm still in the semi-extended position. Sagittal alignment is accomplished through gentle elbow flexion and simultaneous olecranon tip pressure. Hyperflexion of the elbow should be easily achieved without apparent blocks in motion. If after multiple attempts there is persistence of inadequate reduction with residual malalignment, as evidenced by visual, tactile, and fluoroscopic guidance, proceed with open reduction.

\section{Flexion-Type}

For displaced flexion-type fractures, hyperflexion will only lead to worsening of the deformity. Therefore, reduction is best obtained via traction and countertraction with the elbow at 90 degrees of flexion. Fixation following reduction is often staged, with initiation of pin trajectory in the lateral aspect of the distal fragment prior to confirmation of fracture reduction and advancement of pins across fracture site for bicortical purchase.

\section{Open Surgical Technique}

\section{Anterior Approach}

\section{Indications}

- Irreducible extension-type fractures

- Suspected interposition brachialis muscle and fascia

- Median nerve sensory deficits

- Vascular exploration

The anterior approach provides direct access to interposed soft tissue in irreducible extension-type fractures. It is cosmetic when only the transverse portion is utilized but can also be extended to facilitate neurovascular repair when necessary. This approach begins with a 3-4 cm transverse anterior incision over the antecubital fossa, corresponding with the elbow flexion crease and Langerhans lines. Blunt dissection at the subcutaneous level can be performed with assistance of hemostats and a freer elevator. The median nerve and brachial artery are identified and protected with vessel-loops. In fractures with posteromedial displacement in which the radial nerve is at risk, the radial nerve should also be identified between the brachialis and brachioradialis at the lateral aspect of the wound and protected before attempting fracture reduction. Often in the setting of a displaced fracture, the median nerve and brachial artery are not easily identified at the fracture site. If this occurs, these structures should be identified more proximal to the zone of injury, medial to the biceps muscle, and traced distally to the fracture site. Even in the absence of vascular compromise, this step should not be omitted.

Subcutaneous dissection should proceed until the anterior proximal metaphyseal spike is encountered. This is often tethered by a small amount of anterior brachialis fascia with interposition of brachialis fascia, muscle, periosteum and/or capsule at the fracture site. At times, careful extrication of the interposed brachialis fascia bundle and capsule may deliver and untether adjacent neurovascular structures. This can be gently achieved with combination of mosquitos and a freer elevator.

Deep dissection is typically executed at time of injury by the metaphyseal fragment with associated periosteal disruption. Additional fracture site preparation may include gentle irrigation and evacuation of hematoma. Care should be taken to maintain any existing periosteum intact unless there is a clear hindrance to anatomic reduction to promote fracture stability and healing. With posterolateral extension-type fractures, it may be anticipated that anterior and medial periosteum is disrupted. Fracture reduction may proceed under direct visualization and manipulation, and pinning performed percutaneously in standard fashion with fluoroscopic guidance. At our institution, our preference for pin diameter include 0.062 -in $(1.6 \mathrm{~mm}) \mathrm{K}$ wires in patients $\leq 20 \mathrm{~kg}$ and 5/64-in $(2.0 \mathrm{~mm})$ Steinmann pins in patients $>20 \mathrm{~kg} .{ }^{25,26}$ If a vascular injury is identified, and flow to the hand is compromised, vascular repair or reconstruction may commence pending fracture reduction and stabilization. 


\section{Extensile Approach}

The transverse anterior incision may be extensile proximal or distal, medial or lateral, depending on displacement of the distal fragment or vascular exploration needs.

\section{Medial Approach}

\section{Indications}

- Flexion-type fractures

- Ulnar nerve entrapment

- Extension-type fractures with posterolateral displacement

- Oblique or unstable fractures necessitating medial pinning

Depending on the indication for a medial approach, a 3-4 cm longitudinal incision is created over the medial epicondyle. For most cases, a slight posteromedial bias is desirable to provide direct visualization and access to the ulnar nerve. Modifications to this incision may include a slightly shorter incision for medial pinning, centered over the medial epicondyle for medial column trajectory. Blunt dissection through the subcutaneous tissue should be performed to protect the medial antebrachial cutaneous nerve branches in the area. The flexor pronator mass and medial epicondyle will be visible. Once the ulnar nerve has been identified, directly posterior to the medial epicondyle, it should be carefully protected throughout the remainder of the case. We do not advocate for routine dissection of the ulnar nerve. The median nerve and brachial artery will not necessarily be visible in this approach, so only blunt dissection should be performed laterally.

Prior to fracture reduction, hematoma and any interposed soft tissue should be evacuated including the ulnar nerve. Typically, once the hematoma is evacuated and the medial spike of the metaphysis is cleared of brachialis muscle and fascia, reduction is straightforward. For flexion-type fractures, fracture reduction may be aided by traction/counter-traction at the humerus and forearm with elbow flexed no more than 90 degrees and a sterile towel bolster under the olecranon. Standard lateral pin fixation may be performed for biomechanical stability.
When an open reduction is not required but a medial pin is desired, a small incision can be made in the tendinous region of the flexor pronator mass to provide a secure starting point for a medial pin. Additional strategies to mitigate iatrogenic risk to the ulnar nerve include meticulous use of a soft tissue guide or retractor, direct visualization and protection of the nerve when possible, and pin placement with elbow slightly extended.

\section{Postoperative Considerations and Outcomes}

Immediately postoperative to an open reduction, the elbow can be immobilized in 60 to 90 degrees of flexion. Flexion beyond 90 degrees is not necessary as the fracture should be sufficiently stable with internal fixation and will inadvertently increase risk of compartment syndrome. Following open reduction, we recommend overnight versus prolonged day admission to facilitate safe oversight of patient status. This includes serial neurovascular exams, continuous pulse oximetry as an indirect measure of vascular status, and avoidance of narcotic regimen that may mask features of compartment syndrome. Once the surgeon and family can be assured of no evolving or impending compartment syndrome as evidenced by a stable neurovascular exam and comfortable child, the patient can be discharged home. The family should be counseled about the possibility of a delayed presentation of compartment syndrome and effectively educated to recognize potential signs and symptoms (increased pain, increased anxiety, progressive analgesic requirements, agitation). Delayed manifestation of compartment syndrome ( $>12$ hours postoperative) should be specifically considered in children with any compromise of blood flow to the hand post-injury due to the risks of reperfusion syndrome and subsequent limb swelling once flow has been restored. ${ }^{27,28}$ Patients who undergo vascular repair and grafting are typically monitored in the hospital for 48-72 hours after surgery to watch for any changes in vascular status.

While supracondylar fractures reliably heal in 3 weeks, open surgery may delay healing by the necessary stripping of the periosteum. Our standard practice is to leave pins in place for 4 weeks after open reduction. 
Beyond 4 weeks, pin retention may inadvertently lead to pin site issues and is therefore not routinely recommended. The patient can then start gentle range of motion, followed by activities as tolerated; formal physical therapy is usually not necessary and remains controversial in children naturally inclined to free play and self-progression of activity. Return to full activity typically occurs by 6 to 8 weeks postoperatively. Although rare, some loss of full extension has been reported with open reduction in the early postoperative period at a rate of $15 \%$ related to soft tissue disruption, incisional hypertrophy, malunion, and posttraumatic remodeling. ${ }^{19}$ Isolated radial nerve injuries and multiple nerve injuries may further delay motor recovery, with the majority of neurologic deficits anticipated to recover within 6 months without acute nerve decompression. ${ }^{29}$

For those patients who have undergone vascular repair, we recommend long-term follow-up due to the risk of vascular occlusion ${ }^{30}$ as well as avascular necrosis of the trochlea. ${ }^{31}$ Doppler ultrasound can be used to follow the patency of the brachial artery, with overall reassuring patency rates ${ }^{32,33}$ and functional outcomes ${ }^{31,34}$ at intermediate and longer term follow-up to support early open vascular exploration and intervention when indicated. We recommend both radiographs and Doppler ultrasound at 1 year after surgery in these patients, and certainly there are patients who should be monitored even longer.

\section{References}

1. Mitchelson AJ, Illingworth KD, Robinson BS, et al. Patient demographics and risk factors in pediatric distal humeral supracondylar fractures. Orthop Thorofare NJ. 2013;36(6):e700-e706. doi: https://doi. org/10.3928/01477447-20130523-12.

2. Rockwood CA, Beaty JH, Kasser JR. In: Beaty JH, Kasser JR, eds; Skaggs DL, Flynn JM, Waters PM, associate eds. Rockwood and Wilkins' Fractures in Children. 7th edn. Wolters Kluwer/Lippincott Williams \& Wilkins; 2010

3. Cheng JCY, Shen WY. Limb fracture pattern in different pediatric age groups: a study of 3,350 children. J Orthop Trauma. 1993;7(1):5-22. doi: https://doi.org/10.1097/00005131-199302000-00004.

4. Holt JB, Glass NA, Shah AS. Understanding the epidemiology of pediatric supracondylar humeral fractures in the United States: identifying opportunities for intervention. J Pediatr Orthop. 2018;38(5):e245-e251. doi: https://doi.org/10.1097/BPO.0000000000001154.

5. DeFrancesco CJ, Shah AS, Brusalis CM, et al. Rate of open reduction for supracondylar humerus fractures varies across pediatric orthopaedic surgeons: a single-institution analysis. J Orthop Trauma. 2018;32(10):e400-e407. doi: https://doi.org/10.1097/ BOT.0000000000001262.
6. Reitman RD, Waters $\mathrm{P}$, Millis M. Open reduction and internal fixation for supracondylar humerus fractures in children. J Pediatr Orthop. 2001;21(2):157-161. doi: https://doi. org/10.1097/01241398-200103000-00004.

7. Eren A, Güven M, Erol B, et al. Delayed surgical treatment of supracondylar humerus fractures in children using a medial approach. J Child Orthop. 2008;2(1):21-27. doi: https://doi.org/10.1007/ s11832-007-0072-y.

8. Mangat KS, Martin AG, Bache CE. The "pulseless pink" hand after supracondylar fracture of the humerus in children: the predictive value of nerve palsy. J Bone Joint Surg Br. 2009;91(11):1521-1525. doi: https://doi.org/10.1302/0301-620X.91B11.22486.

9. Kuoppala E, Parviainen R, Pokka T, et al. Low incidence of flexiontype supracondylar humerus fractures but high rate of complications: a population-based study during 2000-2009. Acta Orthop. 2016;87(4) 406-411. doi: https://doi.org/10.1080/17453674.2016.1176825.

10. Mahan ST, May CD, Kocher MS. Operative management of displaced flexion supracondylar humerus fractures in children. J Pediatr Orthop. 2007;27(5):551-556. doi: https://doi.org/10.1097/01. bpb.0000279032.04892.6c.

11. Flynn K, Shah AS, Brusalis CM, et al. Flexion-type supracondylar humeral fractures: ulnar nerve injury increases risk of open reduction. $J$ Bone Joint Surg Am. 2017;99(17):1485-1487. doi: https://doi.org/10.2106/ JBJS.17.00068

12. Erez O, Khalil JG, Legakis JE, et al. Ultrasound evaluation of ulnar nerve anatomy in the pediatric population. J Pediatr Orthop. 2012;32(6):641646. doi: https://doi.org/10.1097/BPO.0b013e318263a3c0.

13. Campbell CC, Waters PM, Emans JB, et al. Neurovascular injury and displacement in type iii supracondylar humerus fractures. J Pediatr Orthop. 1995;15(1):47-52. doi: https://doi. org/10.1097/01241398-199501000-00011.

14. Babal JC, Mehlman CT, Klein G. Nerve injuries associated with pediatric supracondylar humeral fractures: a meta-analysis. $J$ Pediatr Orthop. 2010;30(3):253-263. doi: https://doi.org/10.1097/ BPO.0b013e3181d213a6.

15. Armstrong DG, Monahan K, Lehman EB, et al. The pediatric open supracondylar fracture: associated injuries and surgical management. J Pediatr Orthop. 2021;41(4):e342-e346. doi: https://doi.org/10.1097/ BPO.0000000000001772.

16. Nordin A, Shi J, Kenney B, et al. Pediatric supracondylar humerus fractures and vascular injuries: a cross-sectional study based on the National Trauma Data Bank. J Clin Orthop Trauma. 2020;11(2):264-268. doi: https://doi.org/10.1016/j.jcot.2020.01.004.

17. Ozkul E, Gem M, Arslan H, et al. Surgical treatment outcome for open supracondylar humerus fractures in children. Acta Orthop Belg. 2013;79(5):509-513.

18. Özkoc G, Gonc U, Kayaalp A, et al. Displaced supracondylar humeral fractures in children: open reduction vs. closed reduction and pinning. Arch Orthop Trauma Surg. 2004;124(8):547-551. doi: https://doi. org/10.1007/s00402-004-0730-1.

19. Yaokreh JB, Gicquel P, Schneider L, et al. Compared outcomes after percutaneous pinning versus open reduction in paediatric supracondylar elbow fractures. Orthop Traumatol Surg Res. 2012;98(6):645-651. doi: https://doi.org/10.1016/j.otsr.2012.03.021.

20. Koudstaal MJ, de Ridder VA, de Lange S, et al. Pediatric supracondylar humerus fractures: the anterior approach. J Orthop Trauma. 2002;16(6): 409-412. doi: https://doi.org/10.1097/00005131-200207000-00007.

21. Shaw BA, Kasser JR, Emans JB, et al. Management of vascular injuries in displaced supracondylar humerus fractures without arteriography. J Orthop Trauma. 1990;4(1):25-29. doi: https://doi. org/10.1097/00005131-199003000-00004.

22. Shah AS, Waters PM, Bae DS. Treatment of the "pink pulseless hand" in pediatric supracondylar humerus fractures. J Hand Surg Am Ed. 2013;38(7):1399-1403. doi: https://doi.org/10.1016/j.jhsa.2013.03.047. 
23. Badkoobehi H, Choi PD, Bae DS, et al. Management of the pulseless pediatric supracondylar humeral fracture. J Bone Joint Surg Am. 2015;97(11):937-943. doi: https://doi.org/10.2106/JBJS.N.00983.

24. Scannell BP, Brighton BK, VanderHave KL. Neurological and vascular complications associated with supracondylar humeral fractures in children. JBJS Rev. 2015;3(12):1-10. doi: https://doi.org/10.2106/JBJS. RVW.N.00084.

25. Kocher MS, Kasser JR, Waters PM, et al. Lateral entry compared with medial and lateral entry pin fixation for completely displaced supracondylar humeral fractures in children: a randomized clinical trial. J Bone Joint Surg Am. 2007;89(4):706-712. doi: https://doi.org/10.2106/ JBJS.F.00379.

26. Yen YM, Kocher MS. Lateral entry compared with medial and lateral entry pin fixation for completely displaced supracondylar humeral fractures in children: surgical technique. J Bone Joint Surg Am. 2008;90(Suppl_2 Part 1 Suppl 2):20-30. doi: https://doi.org/10.2106/JBJS.G.01337.

27. Ramachandran M, Skaggs DL, Crawford HA, et al. Delaying treatment of supracondylar fractures in children: has the pendulum swung too far? J Bone Joint Surg Br. 2008;90(9):1228-1233. doi: https://doi. org/10.1302/0301-620X.90B9.20728.

28. Cambon-Binder A, Jehanno P, Tribout L, et al. Pulseless supracondylar humeral fractures in children: vascular complications in a ten year series. Int Orthop. 2017;42(4):891-899. doi: https://doi.org/10.1007/ s00264-017-3698-5
29. Shore BJ, Gillespie BT, Miller PE, et al. Recovery of motor nerve injuries associated with displaced, extension-type pediatric supracondylar humerus fractures. J Pediatr Orthop. 2019;39(9):e652-e656.

30. Konstantiniuk P, Fritz G, Ott T, et al. Long-term follow-up of vascular reconstructions after supracondylar humerus fracture with vascular lesion in childhood. Eur J Vasc Endovasc Surg. 2011;42(5):684-688. doi: https:// doi.org/10.1016/j.ejvs.2011.06.028.

31. Scannell BP, Jackson JB, Bray C, et al. The perfused, pulseless supracondylar humeral fracture: intermediate-term follow-up of vascular status and function. J Bone Joint Surg Am. 2013;95(21):1913-1919. doi: https://doi.org/10.2106/JBJS.L.01584.

32. White L, Mehlman CT, Crawford AH. Perfused, pulseless, and puzzling: a systematic review of vascular injuries in pediatric supracondylar humerus fractures and results of a POSNA questionnaire. J Pediatr Orthop. 2010;30(4):328-335. doi: https://doi.org/10.1097/ BPO.0b013e3181da0452.

33. Grant C, Theiss M, Mukherjee D. Pediatric brachial artery injury due to supracondylar humerus fractures: a case series. Vascular. 2019;27(4):451453. doi: https://doi.org/10.1177/1708538119851664.

34. Reigstad O, Thorkildsen R, Grimsgaard C, et al. Supracondylar fractures with circulatory failure after reduction, pinning, and entrapment of the brachial artery: excellent results more than 1 year after open exploration and revascularization. J Orthop Trauma. 2011;25(1):26-30. doi: https:// doi.org/10.1097/BOT.0b013e3181db276a. 